\title{
Challenges in controlling SARS-CoV-2 in a lower-middle income country and the potential unintended effects due to aggressive restrictions
}

Lincoln Leehang Lau* ${ }^{* 1,2,3}$, Peng $\mathrm{Wu}^{4}$, Daryn Joy $\mathrm{Go}^{1}$, Warren Dodd ${ }^{3}$, Charles $\mathrm{Yu}^{5}$

1. International Care Ministries Inc, Manila, Philippines

2. Dalla Lana School of Public Health, University of Toronto, Toronto, Canada

3. School of Public Health and Health Systems, University of Waterloo, Waterloo, Canada

4. WHO Collaborating Centre for Infectious Disease Epidemiology and Control, School of Public Health, Li Ka Shing Faculty of Medicine, University of Hong Kong, Hong Kong SAR, China

5. De La Salle Medical \& Health Sciences Institute, Dasmariñas, Cavite, Philippines

* corresponding author

Lincoln Leehang Lau

International Care Ministries Inc, Philippines

Unit 2001 Antel Global Corporate Center

Julia Vargas Ave, Ortigas Center,

Pasig City, NCR, Philippines

lincoln.lau@caremin.com / lincoln.lau@utoronto.ca

+63998 8489327 


\begin{abstract}
In response to the COVID-19 pandemic, the Philippines placed the majority of the country under enhanced community quarantine, restricting the movement of most of its 100 million plus population. These aggressive measures were initiated on March 15, 2020 and intensified on March 17. According to official data, the number of confirmed COVID-19 cases has exponentially increased during this period, but it is important to note that the number of patients tested also substantially increased during the same period. It is not conclusive that widespread transmission of COVID-19 only started in March and our analysis suggests that community transmission was happening earlier. In discussing extended quarantine measures, it is important to properly understand the trends and recognize the limitations of the data. The unintended consequences on the population, especially in lower-middle income countries with fragile health systems like the Philippines, must be carefully considered.
\end{abstract}

\title{
Background
}

An editorial on the risk South Asia faces in light of SARS-CoV-2 reported that urgent and unified action is needed to avoid a public health catastrophe. ${ }^{1}$ An analysis of national preparedness capacities in light of SARSCoV-2 ended with the stark reality that half of the countries analyzed do not have the adequate capacity to manage an emergency. ${ }^{2}$ The Philippines is a country in Southeast Asia that has recently experienced rapid economic growth, pushing to be re-classified as an upper-middle income country. However, the lack of readiness to respond to a public health emergency like the COVID-19 pandemic has placed the country and fragile healthcare system at risk.

The first case of COVID-19 in the Philippines was confirmed on January 30, 2020. This or other importations likely spread into the community by February. A steady growth in unrecognised infections became apparent in early March when an increasing number of cases with serious illness were recognised. In response, Metro Manila was placed under 'community quarantine' on March 15, which was expanded into a 'lockdown' for the whole island of Luzon (48 million people) two days later. This aggressive restriction of movement was the first for any lower-middle income country and was rapidly replicated in other provinces of the Philippines.

According to data published by the Philippine Department of Health ${ }^{3}$ and the $\mathrm{WHO},{ }^{4}$ the increase in confirmed COVID-19 cases appears to follow the increase in number of patients tested, and vice versa (Figure panel A). While the number of positive cases is clearly increasing exponentially (Figure panel B), this sharp increase might be misinterpreted as evidence of a sudden rise in the transmission of COVID-19 across the country, specifically from March 18 to March 31 when confirmed cases rose from 202 to 2,084. Transmission had likely been occurring for longer but was not realized due to limited testing, which also rapidly increased after March 18. Reports of tourists testing positive for COVID-19 in their home countries following prolonged stays in the Philippines provides indirect evidence of local transmission prior to March 18. Until March 24, 2020, the Philippines had tested fewer than 17 people per million, far fewer than most countries. 

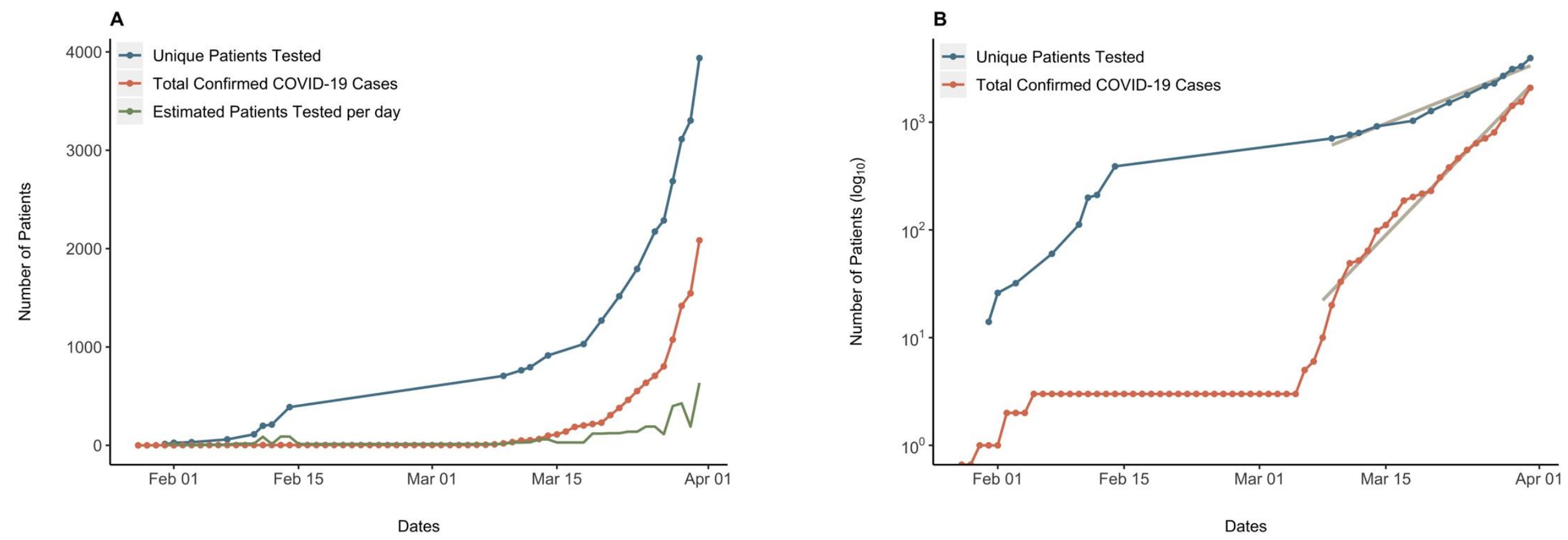

Figure. Confirmed COVID-19 cases and patient testing over time in the Philippines

Trend lines show the progression of confirmed COVID-19 cases (red lines) and number of patients tested (blue lines) on a linear scale (A) and logarithmic scale (B). Number

of patients tested per day is estimated on the linear scale (green line) and straight lines were added to approximate the exponential growth phase. 


\section{Challenges in controlling spread}

Case-based approaches are not feasible without the resources for mass testing. Infrastructure and personnel are necessary for isolating suspected and confirmed COVID-19 cases. In-depth contract tracing requires strong epidemiological surveillance units, which could be why strict quarantine measures were chosen. Encouraging social distancing is challenging when more than $75 \%$ of the economy consists of service sector jobs and industries such as food processing.

Properly interpreting the trends, and recognizing the limitations of data is critical, especially in the context of weaker health systems. Social distancing and quarantine measures will be vital to slow the spread of COVID-19, but we should avoid panic in response to recent increases in cases detected. Appropriate enforcement of restricting the movement of people should be implemented with consideration for households that are in need of food assistance or have no means of income. Recent scenes of people crowding for food dispersals have defeated the purpose of social distancing.

\section{Concerns about domino effects}

Comparable to countries in Africa with weaker health systems, ${ }^{5}$ the Philippines is in a precarious situation. Following outbreaks of dengue, diphtheria, measles and polio in $2019^{6}$ the challenge of mounting disease control campaigns in 2020 will be increasingly difficult. ${ }^{7}$ Under stringent 'lockdown' measures, there is concern for day labourers and service employees without fixed incomes. Although refugee camps are not located in the Philippines, the presence of overcrowded urban slums and busy markets are shared with Bangladesh, and the need for public health systems strengthening is also shared. ${ }^{8}$ Malnutrition in these low income populations is high, $19.9 \%$ of children under five were underweight in one Philippine setting, ${ }^{9}$ which will be further exacerbated if food assistance in not provided.

\section{Conclusions}

In lower-middle income countries the capacity to detect and respond to COVID-19 must be urgently enhanced in order to provide accurate data for public health officials and the government. In light of the challenges and the possibility of unintended effects, implementing or extending strict quarantine measures must be carefully considered. Mitigation strategies such as public health campaigns to encourage increased hygiene, mask use, social distancing, and banning mass gatherings are options but difficult to implement. The private sector and large corporations should also play a role by supporting vulnerable employees with flexible employment during this time. Increasing the frequency of public transport while limiting occupancy could be considered, as well as makeshift hospitals to treat mild cases, removing them from the community. Telehealth or internet-based assessment and care options for mild cases could help decongest hospitals and health providers. While these mitigation and support measures might diverge from the stringent 'lockdown' and other resource intensive strategies that can be implemented in Europe and North America, ${ }^{10}$ the potential unintended impact on health as a whole in lower-middle income countries should closely monitored as the situation continues to develop. 


\section{Acknowledgements}

The authors thank Hannah Ferrolino and Mia Choi for data collection, and Ben Cowling for helpful discussions.

\section{References}

1 Bhutta ZA, Basnyat B, Saha S, Laxminarayan R. Covid-19 risks and response in South Asia. Bmj 2020; 368: $\mathrm{m} 1190$.

2 Kandel N, Chungong S, Omaar A, Xing J. Health security capacities in the context of COVID-19 outbreak: an analysis of International Health Regulations annual report data from 182 countries. Lancet 2020; 6736: $1-7$.

3 Republic of the Philippines Department of Health. 2019-nCoV Daily Situation Reports. 2020 https://www.doh.gov.ph/2019-nCov/situational-report.

4 World Health Organization. Coronavirus disease (COVID-19) situations reports in the Philippines. 2020 https://www.who.int/philippines/emergencies/covid-19-in-the-philippines/covid-19-sitreps-philippines.

5 Martinez-Alvarez M, Jarde A, Usuf E, et al. COVID-19 pandemic in west Africa. Lancet Glob Heal 2020; 2019: 2019-20.

$6 \quad$ The Lancet Infectious Diseases. Infectious disease crisis in the Philippines. Lancet Infect Dis 2019; 19: 1265.

$7 \quad$ Roberts L. 'We have no choice.' Pandemic forces polio eradication groups to halt campaigns. Sci. Mag. News. 2020; published online April 1. DOI:10.1126/science.abc0263.

$8 \quad$ Vince G. The world's largest refugee camp prepares for covid-19. Bmj 2020; 368: m1205.

9 Capanzana M V., Aguila D V., Gironella GMP, Montecillo K V. Nutritional status of children ages 0-5 and 5-10 years old in households headed by fisherfolks in the Philippines. Arch Public Heal 2018; 76: $1-8$.

10 Fineberg H V. Ten Weeks to Crush the Curve. N Engl J Med 2020; : 1-2.

\section{Data Availability}

All data used in this study is available from the corresponding author upon request.

\section{Funding}

No funding was received to produce this study.

\section{Author Contributions}

LLL, PW, and DJG designed the study. LLL and DJG analyzed the data. LLL, WP, DJG, WD and CY interpreted the data, and contributed to the final manuscript. All authors have reviewed and approved the manuscript.

\section{Competing Interests}

All authors have completed the ICMJE form for disclosure of potential conflicts of interest and all authors on all 6 parts have nothing to disclose. 